\title{
Contribuições do PET-Saúde/Redes de Atenção Psicossocial à Saúde da Família
}

\author{
Larissa de Almeida Rézio(a) \\ Thais Nagano Moro ${ }^{(b)}$ \\ Samira Reschetti Marcon ${ }^{(c)}$ \\ Cinira Magali Fortuna ${ }^{(d)}$
}

Rézio LA, Moro TN, Marcon SR, Fortuna CM. Contributions of the PET-Health/Psychosocial Healthcare Networks to Family Healthcare. Interface (Botucatu). 2015;19 Supl 1:793-804.

This study aimed to analyze the contributions of the PET-Health/ Psychosocial Health Care Networks to a Family Health Strategy Unit in Cuiaba, Mato Grosso, Brazil. This was a descriptive study employing a qualitative approach by interviews with five health professionals and two scholarship students as well as the records of activities conducted in the Health Service. Through thematic content, four sub-themes were systematized: "the network connection," "qualification of mental healthcare," "improvement of professional knowledge," and "overcoming stigmas about patients in psychological suffering." The result suggested that there was sensitization of professionals and scholarship students with regard to the importance of monitoring people with mental distress in primary care in coordination with mental health services.

Keywords: Mental Health. Staff Development. Primary Healthcare. Health Personnel. Education by Work for Health Program.
O estudo objetivou analisar as contribuições do Programa de Educação pelo Trabalho para a Saúde - PET-Saúde/Redes de Atenção Psicossocial à Saúde - a uma unidade de Estratégia de Saúde da Família em Cuiabá, Mato Grosso, Brasil. Trata-se de um estudo descritivo, com abordagem qualitativa, utilizando entrevista com cinco profissionais de saúde e dois acadêmicos bolsistas e registros de atividades realizadas no serviço. À luz da análise de conteúdo temática, sistematizamos quatro subtemas: "articulação da rede"; " qualificação da assistência à saúde mental", "melhora do conhecimento dos profissionais" e "superação de estigmas sobre o paciente em sofrimento psíquico". Houve sensibilização dos profissionais e bolsistas sobre a importância do acompanhamento das pessoas com sofrimento mental na atenção básica, em articulação com serviços de saúde mental.

Palavras-chave: Saúde Mental. Desenvolvimento de Pessoal. Atenção Básica à Saúde. Pessoal de Saúde. Programa de Educação pelo Trabalho para a Saúde. $(a, c)$ Faculdade de Enfermagem, Universidade Federal de Mato Grosso. Av. Fernando Corrêa da Costa, $n^{\circ} 2367$. Bairro Boa Esperança. Cuiabá, MT, Brasil. 78060-900 larissarezio@hotmail.com; samira.marcon@gmail.com

(b) Hospital Santa Rosa. Cuiabá, MT, Brasil. thais.n.moro@gmail.com

(d) Departamento Materno-Infantil e Saúde Pública, Escola de Enfermagem de Ribeirão Preto, Universidade de São Paulo. Ribeirão Preto, SP, Brasil. fortuna@eerp.usp.br 


\section{Introdução}

Diante da evidência das precárias condições em que viviam as pessoas em sofrimento psíquico no mundo e no Brasil, a partir do século XIX, foram desencadeados, mundialmente, movimentos contrariando o modelo psiquiátrico vigente. No Brasil, entre os anos 1970 e 80, o movimento de mudança denominado Reforma Psiquiátrica se fortaleceu influenciado, sobretudo, pela experiência italiana, por meio dos Centros de Saúde Mental, que assumiam o cuidado integral em saúde mental e a reconstrução das formas como a sociedade lidava com o paciente em sofrimento psíquico ${ }^{1}$, facilitando, dessa forma, a inclusão social.

Embora a Reforma Psiquiátrica seja um movimento com foco na reinserção social e desinstitucionalização dos usuários ${ }^{1}$, ainda existem obstáculos para a superação da hegemonia do modelo médico-psiquiátrico e sua efetivação, como, por exemplo: o tempo de tramitação da Lei no 10.216, que dispõe sobre a proteção e os direitos das pessoas portadoras de transtornos mentais, aprovada, apenas, em 06 de abril de 2001²; assim como, a criação de legislações que amparem a criação de serviços especializados, como a Portaria Reguladora dos Centros de Atenção Psicossocial (Caps), aprovada somente em 2002.

Em Mato Grosso, a trajetória da Reforma Psiquiátrica teve um retardo em relação a outros estados brasileiros, tendo a sua Política Estadual de Saúde Mental regimentada e aprovada, apenas, em dezembro de 2011 (Resolução n 14/2011) - muito tardiamente, se comparada ao movimento da Reforma Psiquiátrica que ocorreu na década de 1980 no restante do Brasil ${ }^{3}$.

Nesse processo de transformação legislativa e política, o Ministério da Saúde (MS), em parceria com o Ministério da Educação, criou o Programa de Educação pelo Trabalho para a Saúde (PET Saúde), regulamentado pela Portaria Interministerial no 421, de 3 de março de 2010. Esse programa busca a integração ensino-serviço-comunidade, e objetiva: o incentivo à formação do discente com perfil adequado para atuar nos serviços do Sistema Único de Saúde (SUS); a inserção/articulação da academia nos serviços, e a qualificação de profissionais e docentes ${ }^{4}$.

Nesta lógica, em março de 2013, foi lançado o PET-Saúde/Redes de Atenção à Saúde (PET/Redes), que busca o desenvolvimento de intervenções no modelo de rede, com vistas a qualificar as ações e os diferentes serviços de saúde oferecidos à população ${ }^{5}$. Especificamente na atenção à saúde mental, dentre as diferentes configurações em Rede, foi proposto o PET/Redes de Atenção Psicossocial (PET-RAPS), pautado na proposta da Rede de Atenção Psicossocial (RAPS), pactuada na reunião da Comissão Intergestores Tripartite de 24 de novembro de 2011. A RAPS procura garantir a atenção às pessoas em sofrimento mental e as necessidades decorrentes do uso de crack, álcool e outras drogas, com foco na efetivação do cuidado integral, considerando as dimensões biológicas, psicológicas e sociais ${ }^{6}$.

A constituição da Rede possibilita a superação de dificuldades na articulação efetiva entre a Estratégia de Saúde da Família (ESF) e os serviços de saúde mental/ CAPS. Tais dificuldades decorrem de: escassez de pessoal qualificado, formação profissional incipiente em saúde mental, desarticulação dos serviços e o trabalho fragmentado, não-valorização e qualificação da escuta e do acolhimento do sujeito em sofrimento psíquico, falta de apoio matricial, ferramenta importante de aproximação entre ESF e CAPS, além de práticas vinculadas ao modelo biomédico e o estigma que permeia o campo da saúde mental7-9.

Deste modo, a criação do PET-RAPS é uma estratégia importante para a efetivação da reforma psiquiátrica, para a qualificação do cuidado e para a articulação da RAPS de modo geral, com enfoque na atenção básica, considerando que este é um espaço importante de acolhimento e proximidade, além de ser de fácil acesso à população ${ }^{10}$. Ressalta-se, ainda, que a atenção básica também constitui um local importante para o desenvolvimento de atividades de extensão por instituições formadoras, fortalecendo a relação ensino-serviço-comunidade ${ }^{10}$ e formando trabalhadores para esse nível de atenção.

A partir do exposto, compreendendo a importância da constituição da RAPS no município de Cuiabá-MT, assim como, a efetivação de práticas de saúde mental na atenção básica que acolham o usuário integralmente, objetivamos analisar as contribuições do PET-RAPS em uma Unidade de Estratégia de Saúde da Família do município de Cuiabá-MT. 


\section{Metodologia}

Essa é uma pesquisa de natureza exploratória e descritiva, de abordagem qualitativa, desenvolvida em uma ESF do município de Cuiabá-MT, no primeiro semestre de 2014.

A escolha do serviço se deu por ser, esse, um espaço onde têm sido desenvolvida, há mais de seis meses, extensão universitária, por meio do PET-RAPS, pela Faculdade de Enfermagem da Universidade Federal de Mato Grosso (UFMT). Ao iniciarmos as atividades do Programa, utilizamos a metodologia da problematização, com a qual, ao realizarmos o levantamento de problemas, evidenciamos que não havia ações de saúde mental nesta unidade. Deste modo, o PET-RAPS foi responsável pela indução: de um diagnóstico coletivo e implantação da ficha de busca ativa, de estudo de caso, de reuniões conjuntas entre ESF e CAPS, discussão de textos, visitas domiciliares de acompanhamento em saúde mental, e implantação da ficha de acompanhamento dos usuários ao serviço.

Para que o desenvolvimento do programa fosse efetivo, foram realizados encontros quinzenais entre tutores (docentes da UFMT), discentes bolsistas de diferentes cursos, e preceptores (profissionais dos serviços), para discussão e avaliação das ações realizadas.

Desse modo, a pesquisa emergiu da necessidade de se analisar a parceria PET-RAPS e os serviços envolvidos.

A população de estudo foi constituída por: cinco profissionais da equipe da ESF (uma enfermeira, um médico, duas agentes comunitárias de saúde e uma técnica de enfermagem), e duas discentes bolsistas do PET-RAPS, dos cursos de Enfermagem e Psicologia da UFMT. Foram incluídos todos os profissionais que estiveram no serviço durante os últimos seis meses, independente do tipo de vínculo empregatício, e as bolsistas vinculadas ao PET. Esse período foi definido considerando-se o início das intervenções do projeto no serviço.

O material empírico constituiu-se das entrevistas transcritas dos profissionais e bolsistas, e de livros de registro contendo informações acerca de todas as atividades desenvolvidas pelo PET, ou seja: atendimentos, visitas domiciliares, discussão de textos, participação em alguma ação do serviço, dentre outras.

Utilizamos, como instrumento de coleta de dados, um roteiro de entrevista semiestruturada, com questões relacionadas: à inserção do PET na Unidade e suas ações, a articulação da RAPS, e a incorporação da saúde mental na atenção básica. As entrevistas foram realizadas em ambiente reservado, com duração média de uma hora. Ainda como fonte de dados, foram verificados os livros de registros das atividades diárias dos bolsistas e preceptor/profissionais vinculados ao PET, em que utilizamos um roteiro direcionado para as ações de saúde mental realizadas pelos bolsistas e suas respectivas contribuições para o serviço.

Para análise dos dados, utilizamos a técnica de análise de conteúdo do tipo temática, originando temas e subtemas. A análise de conteúdo é uma técnica de análise das comunicações por meio de procedimentos objetivos e sistemáticos, podendo ser qualitativos ou quantitativos, que permitem a inferência de conhecimento relativo às condições de produção dessas mensagens. Neste tipo de análise, é possível encontrar os núcleos de sentido das mensagens ${ }^{11}$.

Após a leitura exaustiva de todos os dados coletados, foi possível encontrar quatro subtemas: Articulação da rede; Qualificação da assistência à saúde mental; Melhora do conhecimento dos profissionais e Superação de estigmas sobre o paciente em sofrimento psíquico. A partir dos subtemas, identificamos o tema central, denominado contribuições do PET para a unidade de ESF, o qual foi analisado conforme: literatura disponível referente à RAPS, trabalho em saúde mental, saúde mental na atenção básica, e articulação da RAPS.

Com o objetivo de manter o anonimato dos entrevistados, as falas foram apresentadas no texto com siglas e números, sendo: T (1 a 5) para trabalhadores/ profissionais, B (1 e 2) para as discentes bolsistas do PET, e LR para os dados coletados no livro de registros das atividades diárias das bolsistas e preceptor/profissionais.

Este estudo foi aprovado pelo Comitê de Ética em Pesquisa do Hospital Universitário Júlio Muller, respeitando a Resolução nº 466 do Conselho Nacional de Saúde, de 2012. 


\section{Resultados e discussão}

\section{Contribuições do PET para a equipe da ESF}

\section{Articulação da Rede}

Estudos têm mostrado que, apesar dos esforços do MS em incluir ações de saúde mental na atenção básica, essa realidade ainda é pouco frequente ${ }^{12-14}$, embora seja essencial para efetivação da Reforma Psiquiátrica, assim como para a atenção integral do ser humano.

Entretanto, conforme preconizado pelo MS, a consolidação da Reforma Psiquiátrica depende, necessariamente, da construção de uma rede comunitária de cuidados, com diferentes serviços substitutivos ao modelo hospitalocêntrico, articulados entre si de modo a formar um conjunto de referências com a capacidade de acolher e de cuidar das pessoas em sofrimento psíquico ${ }^{15}$.

Essa articulação em rede supera o conceito de centralidade de cuidados, oferecendo dinamismo e flexibilidade aos serviços, gerando melhores resultados na assistência ${ }^{15}$. A constituição da rede, implica: uma série de pontos de encontros, de trajetórias de cooperação, de simultaneidade de iniciativas e atores sociais envolvidos, pois, nessa lógica de funcionamento, se concretiza a implementação do modelo de atenção psicossocial ${ }^{1}$. As falas abaixo ilustram esse aspecto:

“A articulação só tá existindo hoje, dessa forma, assim bem organizada por causa do PET. [...] A gente teve reuniões com grupos de estudos que são de educação permanente, reuniões de subgrupos, rodas de conversa, articulações entre as redes, entre CAPS e o PSF. [...] O PSF foi para - CAPS para conhecer o local, para ter uma relação direta com os profissionais, para conhecer o serviço, para promover mesmo a articulação entre as redes, e depois em outro momento a equipe do CAPS veio até o PSF para conhecer [...] e aí a gente discutiu os temas juntamente entre as duas equipes". (T1)

"Nos reunimos no CAPS para iniciarmos nossas intervenções. A equipe da ESF estava presente no CAPS juntamente com a equipe de lá. Iniciamos a roda de conversa [...]". (LR)

“Para o usuário proporcionou ele ter um serviço que serve de referência, porque antes não era comum, por exemplo, um paciente esquizofrênico procurar um PSF pra ser atendido". (T4)

Assim como outros estudos que apresentam a necessidade de um trabalho pautado na articulação em Rede, e a realização de um atendimento mais singularizado como meio de aumentar a capacidade resolutiva dos problemas ${ }^{13,14}$, também percebemos que a inserção do PET-RAPS na ESF, foi fator essencial para iniciar o trabalho de corresponsabilização dos casos de saúde mental do território; o que, além de singularizar o tratamento, reduziu os encaminhamentos da ESF para o CAPS, possibilitando o empoderamento dessa equipe para resolução de problemas.

A noção de território não deve se restringir apenas ao espaço geográfico, mas, sim, de pessoas, instituições, redes e cenários onde ocorrem as relações de vida diária de uma dada sociedade ${ }^{1}$. $\mathrm{O}$ território é a área sobre a qual o serviço deve assumir a responsabilidade relacionada às questões de saúde mental ${ }^{16}$; e essas ações proporcionam a construção do papel terapêutico inerente ao vínculo e permitem o acompanhamento qualificado aos usuários pela ESF ${ }^{13}$.

Sobre o papel do CAPS, embora seja um de seus objetivos oferecer suporte em saúde mental à atenção básica, além de supervisionar e capacitar as equipes de saúde da família ${ }^{17}$, essas ações só foram impulsionadas com a implantação do PET-RAPS, conforme evidenciado nas falas acima.

Desse modo, o trabalho interprofissional pode se constituir a partir da possibilidade de encontros de trabalhadores dos serviços de saúde mental e de serviços de atenção básica, sendo o PET-RAPS um 
disparador dos mesmos. Estudo ${ }^{18}$ mostra a importância da formação para o aprendizado do trabalho interprofissional que carece de investimentos, podendo ser favorecida por estratégias que colocam estudantes de diferentes cursos juntos em situações reais de trabalho.

“O programa trouxe essa atividade em relação à saúde mental que antes não tinha [...]. Por que foi através do PET que começou". (T3)

"Realizamos um grupo para discussão da temática rede, juntamente com a equipe do CAPS e ESF, sob coordenação das bolsistas". (LR)

A partir disso, ressaltamos a importância do PET como articulador dos encontros sistematizados entre CAPS e ESF ao longo do desenvolvimento do projeto, proporcionando: não só o conhecimento da localização geográfica do CAPS, mas a aproximação dos profissionais da ESF com a temática de saúde mental, a compreensão desses quanto à finalidade de um serviço de atenção à saúde mental, as atividades oferecidas, assim como, os papéis dos profissionais da equipe interdisciplinar, facilitando, portanto, a articulação da Rede. Também permite, à equipe de saúde mental, compreender o trabalho da equipe de atenção básica em seus desafios.

\section{Qualificação da assistência à saúde mental}

A ESF é um componente essencial para efetivação das ações de saúde mental na atenção básica, pois valoriza os princípios: da territorialização, do vínculo com a população, do trabalho em equipe e da participação democrática, participativa e solidária da comunidade ${ }^{19}$. Essa nova forma de lidar com as pessoas em sofrimento psíquico auxilia no estabelecimento de uma equipe multiprofissional mais preparada e comprometida com os usuários, podendo proporcionar um atendimento mais qualificado.

Nesse contexto de qualificação da assistência, no qual há a incorporação do cuidado em saúde mental na atenção básica, se faz necessário um diagnóstico da população da área de abrangência quanto a possíveis casos de sofrimento psíquico; e, para tal, a ficha de busca ativa pode ser um importante instrumento direcionador. Essa consiste em nove questões relativas a problemas de saúde mental, com linguagem simples, respostas dicotômicas (sim ou não), e pode ser aplicada pela Agente Comunitária de Saúde (ACS) no momento do cadastro das famílias ${ }^{20}$.

A ficha de busca ativa possibilita obter um cadastro das famílias que precisam ser avaliadas, além de ser importante no conjunto de ações em vigilância epidemiológica, de investigação de campo e identificação de pessoas em condições de sofrimento psíquico. Sua utilização permite, também, confirmar casos suspeitos, possibilitando decisões mais adequadas em relação a encaminhamentos e à assistência²1 .

"Não existia a ficha de busca ativa, então a partir do momento que teve a aplicação da ficha da busca ativa nós já tivemos um olhar assim mais amplo né? [...] E a partir daí gente já começa a fazer os acompanhamentos. [...] com estudos de casos voltados para os usuários que a gente acompanha, visitas domiciliares, acompanhamento na unidade e nas casas, atendimento de saúde mental, aplicação da ficha de busca ativa e a ficha de acompanhamento também voltado para saúde mental". (T1)

"Sim, busca ativa, a articulação do PSF com o CAPS e reuniões entre a gente que não tinha antes, e consultas voltadas pra saúde mental também antes não tinha". (T3)

"Foi criada a parte de saúde mental, que as agentes comunitárias fazem a busca ativa na comunidade".

"A gente discute os casos e tem também a busca ativa, mas aí quem faz são as agentes junto com as alunas que também participaram". (T5) 
"Realizamos busca ativa de usuários em sofrimento psíquico com a ACS". (LR)

A inserção do PET no serviço determinou a incorporação da ficha de busca ativa na prática da equipe da ESF; e essa atitude não só possibilitou identificar e cadastrar os usuários que necessitavam de atendimento em saúde mental, como, também, aproximou a equipe da população e auxiliou no acompanhamento dessas pessoas.

Outra estratégia importante para acompanhamento comunitário em saúde mental é a visita domiciliar. Com a sua realização na rotina do serviço, os profissionais da ESF têm a possibilidade de conhecer a real situação das famílias e de seus membros individualmente, dando condições para que sejam efetivadas intervenções que possam ajudar no ambiente familiar, além de essa estratégia auxiliar na construção e fortalecimento do vínculo entre os profissionais e familiares ${ }^{22}$.

"Fomos ao PSF e conversamos com a preceptora e com a ACS sobre o caso de um paciente de saúde mental. Saímos para visita na casa desse paciente e de uma outra paciente, depois voltamos para conversar sobre o caso e planejar ações para intervir". (LR)

"Teve uma vez que uma paciente veio até as meninas [na ESF] [elas] orientaram, ligaram lá no CAPS perguntaram certinho como seria o atendimento e orientaram tudo certinho". (T2)

Observamos que, após a inserção do Programa, bem como das bolsistas na unidade, algumas ações de saúde mental começaram a se efetivar, dentre elas: a visita domiciliar visando não somente evidenciar situações de sofrimento psíquico, mas, frente a essas, a responsabilização pela equipe quanto ao acompanhamento e encaminhamentos necessários para estes usuários.

Embora o relato acima demonstre o olhar do profissional para as necessidades de acompanhamento às pessoas em sofrimento psíquico, se refere a essas como 'usuários de saúde mental', denotando, ainda, a separação entre o físico e o psíquico, como se essas necessidades não fossem únicas e sobrepostas no ser humano. Portanto, a visão integral do usuário e a incorporação dessa prática pelos profissionais ainda precisam ser trabalhadas, tendo o PET papel fundamental neste processo.

A fala nos permite evidenciar que as visitas domiciliares e o acompanhamento dos usuários se intensificaram, e estão sendo incorporados pela equipe de forma contínua.

Outro aspecto importante relacionado à qualificação da assistência é o acolhimento, considerado um recurso terapêutico importante na $\mathrm{ESF}^{8}$, e que deve ser entendido de modo mais amplo do que o empregado na prática tradicional, pois é um recurso que pode garantir o acesso aos serviços de saúde, no qual os mesmos terão responsabilidade com as necessidades de saúde da população atendida ${ }^{23}$.

Entendemos, portanto, que há necessidade de se estabelecerem vínculos afetivos e profissionais com as pessoas em sofrimento psíquico, para que se sintam realmente ouvidas e cuidadas, sintam que os profissionais que as escutam estão efetivamente focados em seus problemas, dispostos a ajudá-las, e a se responsabilizarem pelo seu cuidado ${ }^{1}$. Aspectos estes possibilitados pelo ato de acolher.

"Antes eles [equipe multiprofissional da unidade] tinham um olhar diferente do usuário com transtorno mental e hoje já conseguem fazer um acolhimento de forma mais humanizada, dar as informações mais esclarecidas e assim o paciente fica mais é satisfeito com a unidade". (T4)

Assim, identificamos que a nova forma de agir dos profissionais resultou em mudanças no atendimento e cuidado dos usuários em sofrimento psíquico.

Em consonância com as Políticas Ministeriais ${ }^{23}$, essas ações contribuem para a efetivação da saúde mental na atenção básica, pois possibilitam qualidade da assistência, por meio do acolhimento e aproximação da equipe com os usuários, construindo e fortalecendo os vínculos.

Essas novas práticas são primordiais não apenas para a efetivação dos princípios e diretrizes da Reforma Psiquiátrica, mas, também, para a atenção integral da pessoa em sofrimento psíquico no local que se constitui como porta de entrada para o sistema de saúde - e essas ações ocorreram após 
a inserção do PET-RAPS. Portanto, o programa teve uma contribuição importante no processo de qualificação da assistência.

\section{Melhora do conhecimento dos profissionais}

Além das contribuições referentes à criação de novas práticas no serviço voltadas à saúde mental, estudos têm reafirmado que o programa PET repercute positivamente em relação à qualificação da assistência, ao estímulo dos profissionais a uma prática reflexiva, reorientando o serviço e aumentando a acessibilidade, e, consequentemente, melhorando o acolhimento ${ }^{12,24}$.

Deste modo, considerando que a Reforma psiquiátrica é um movimento social complexo, envolvendo aspetos indissociáveis nas dimensões teórico-conceitual, técnico-assistencial, jurídico-político e sociocultural, entendemos que o PET contribui com aspecto técnico-assistencial, na medida em que amplia a noção de integralidade no campo da saúde mental e atenção psicossocial, e auxilia na construção de dispositivos estratégicos, onde é possível acolher, cuidar, socializar e produzir novas subjetividades ${ }^{1}$.

"A gente fez alguns estudos de caso, a gente estudou a temática voltada pra saúde mental que as tutoras cobraram muito isso da gente e isso é muito bom. Acaba fazendo você buscar o seu autoconhecimento. Porque tem muitas coisas que você deixa a desejar, então você vai sempre estar pesquisando, atualizando seus conhecimentos [...] E também da forma que eu tenho que oferecer uma assistência de qualidade para o usuário, porque se eu to preparada, hoje eu me sinto preparada, lógico que não $100 \%$, porque é no dia a dia a gente vai aprendendo, as coisas vão mudando. Eu já não vou ter mais aquela total insegurança de atender como eu tinha antes né?" (T1)

"Hoje eu percebo que a equipe tem um conhecimento a mais voltado para saúde mental e que foi um grande ganho assim depois da atuação do PET. [...] Proporcionou o aprendizado individual de cada uma delas". (T2)

Diante do exposto, podemos afirmar que o PET auxiliou na formação dos profissionais, estimulando-os à reflexão sobre o seu papel, com ênfase nos aspectos socioculturais, contrariando o modelo tecnicista ${ }^{15,24}$; além de contribuir para o aprendizado individual, com profissionais mais preparados, ativos e críticos de sua aprendizagem, colaborando para a solução de problemas de saúde de forma mais efetiva.

Estudos têm evidenciado que a maioria das ESF apresenta dificuldades em realizar atendimento em saúde mental, não realizando atividades referentes a esta área ${ }^{18,24,25}$; essa também era a realidade dessa equipe, como demonstram as falas a seguir:

"A gente sabia que tinha os usuários com sofrimento mental, mas a gente fazia o acompanhamento assim, quando precisasse de uma renovação de receita, daí o usuário vinha marcava a consulta, renovava a receita e ai deixava o acompanhamento a desejar né?" (T1)

"Sempre que era alguma coisa relacionada à saúde mental busca o antigo Adauto [Hospital Psiquiátrico Estadual de MT] e nada aqui. [...] E agora já tem, nesse sentido de uma resposta aos anseios deles, um lugar onde eles podem vir para conversar". (T3)

Antes do PET-RAPS iniciar na ESF, as ações de saúde mental eram bem limitadas e/ou não ocorriam. Os usuários em sofrimento psíquico não eram acompanhados, e as ações não eram fundamentadas nos princípios da Reforma Psiquiátrica, e sim no modelo biomédico, com foco na medicalização.

Por isso, enfatizamos que a formação de profissionais mais aptos e preparados para atender as pessoas em sofrimento psíquico e o conhecimento adquirido por esses profissionais contribuíram para que o acolhimento da população com demandas de saúde mental se aperfeiçoasse e, consequentemente, também a assistência. 


\section{Superação de estigmas sobre o paciente em sofrimento psíquico}

Embora, pontualmente, a implementação de cuidado em saúde mental na atenção básica esteja sendo realizada, ainda é presente o preconceito às pessoas em sofrimento psíquico na maioria das

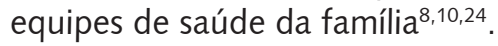

O desafio de atualizar as equipes a pensarem e agirem quebrando os estigmas do modelo biomédico, não é tarefa fácil; entretanto, com o aperfeiçoamento da equipe, o conhecimento e o recurso do acolhimento - ferramentas essas que visam ampliar a capacidade de escuta dos trabalhadores - possibilitam que a equipe, família e usuários possam pensar juntos em intervenções que ajudem a superar as dificuldades presentes ${ }^{13}$.

No tocante à reflexão anteriormente feita sobre a complexidade da Reforma Psiquiátrica, bem como de seus aspectos, é relevante retomarmos o contexto sociocultural, que envolve a desconstrução de estigmas. Esse aspecto tem, como princípio fundamental, o envolvimento da sociedade na discussão da reforma psiquiátrica e da saúde mental, visando à participação social e política de todos os atores envolvidos, objetivando a mudança de mentalidade, atitudes, relação sociais e a construção de reciprocidade e solidariedade ${ }^{1}$.

Portanto, a inclusão das ações de saúde mental na atenção básica possibilita avançar no processo de desinstitucionalização, contribuindo para a desmistificação da loucura ${ }^{16}$, na medida em que se trabalha com a dimensão sociocultural.

"A saúde mental pra mim, antes de ter contato com o PET, antes de ter essas conversas, pra mim era coisas de outro mundo. Dava medo, na hora que o pessoal falava sobre saúde mental. Eu pra mim tinha como um louco né, aí com essas explicações, com essas conversas e reuniões, a gente teve aquela separação da doença, das pessoas assim, como que era a reação né, tudinho". (T5)

"Eu acho muito importante porque a gente teve assim uma diferenciação, porque no começo quando a gente começou a trabalhar tinha um olhar diferente com relação aos pacientes que tinha sofrimento mental e depois que o PET veio pra cá abriu nossos horizontes, a gente está percebendo como tratar esse paciente, como passar uma informação mais correta pra ele, orientar para onde ele possa ir depois daqui da unidade". (T2)

"Agora eu acredito que não só as ACS, mas a enfermeira e o médico têm outro olhar em relação à saúde mental". (T3)

"A gente começou a criar intervenções para a equipe, para tentar fazer com que a equipe olhasse de uma forma totalmente diferente para a saúde mental [...] e tirar assim, desconstruir a ideia de que eles tinham de louco e reconstruir uma ideia de ressocializar essa pessoa, de acolher essa pessoa em sofrimento e eu acho que está mudando". (B1)

Sentimentos como medo da loucura e agressividade foram trabalhados, na medida em que o PET contribuiu para a desconstrução de estigmas e preconceitos relacionados à pessoa em sofrimento psíquico por meio de discussões com a equipe. Desta forma, também contribuiu para a implementação do modelo de atenção psicossocial, ao passo que valorizou os aspectos socioculturais.

Com a problematização de estereótipos sobre a loucura, o PET possibilitou a aproximação dos profissionais às famílias e/ou pessoas em sofrimento psíquico ou dependentes de substâncias psicoativas, superando a referência do modelo biomédico, construindo diariamente novas maneiras de auxiliar os usuários em suas dificuldades, estimulando-os quanto ao próprio cuidado com mais autonomia ${ }^{16}$.

É importante ressaltar que, embora tenha sido desencadeado um processo de desmistificação da "loucura" na unidade pesquisada, e que algumas ações de cuidado à saúde mental começaram a ser incorporadas na prática daqueles profissionais, os serviços de atenção à saúde, isoladamente, não são capazes de construir mudanças significativas, pelo fato da necessidade de articulação em rede. Por isso, são necessárias práticas articuladas entre diferentes saberes e níveis de complexidade na produção do 
cuidado, para que se potencialize e operacionalize a integralidade da atenção, que é necessária para auxiliar na construção de novos rumos para o campo da atenção psicossocial ${ }^{16}$.

Entendemos que o PET-RAPS auxiliou nesse processo de construção, além de possibilitar a problematização dos preconceitos trazidos do modelo hospitalocêntrico, por meio da contribuição referente à formação de profissionais para o trabalho em saúde mental, possibilitando maior segurança e autonomia na assistência.

Diante disso, podemos constatar que a Reforma Psiquiátrica brasileira é um processo em movimento de construção, a partir de reflexões e mudanças que ocorrem, a um só tempo, nos campos assistencial, cultural e conceitual. Assim, esse processo social complexo objetiva mudar as relações que a sociedade, os sujeitos e as instituições estabeleceram com o sofrimento psíquico, conduzindo-as no sentido de superação do estigma, de integração, de estabelecer com a loucura uma relação de coexistência, troca, solidariedade, positividade e de cuidados ${ }^{1}$.

Apesar de o PET-RAPS ter contribuído de várias maneiras para a melhora da assistência aos usuários em sofrimento psíquico, ainda existem desafios para a implementação de ações de saúde mental na atenção básica. Esses desafios estão relacionados, sobretudo, ao despreparo das equipes para lidarem com o paciente em sofrimento psíquico, e à falta de profissionais na equipe, que, em muitas situações, encontra-se em número mínimo, o que influencia diretamente na realização das ações de saúde mental, e, consequentemente, na efetivação da Reforma Psiquiátrica.

Para superar esses desafios, se faz necessário compreender o processo social em toda a sua complexidade, desconstruindo e reconstruindo conceitos fundantes da psiquiatria (campo teórico-conceitual); com a criação de espaços de sociabilidade, trocas e produção de subjetividades (técnico-assistencial); as legislações sanitárias, civil e penal (jurídico-político), e, ainda, transformar o imaginário social, como o campo mais importante de todos (sociocultural) ${ }^{1}$.

\section{Considerações finais}

O PET-RAPS foi importante para desencadear o processo de articulação entre a ESF e o CAPS, estratégia necessária para: a efetivação da Reforma Psiquiátrica, a consolidação da rede e corresponsabilização pelo usuário, em função da aproximação dos profissionais de serviços diferentes, fortalecendo a importância da inclusão e da execução de ações de saúde mental na atenção básica.

O desenvolvimento do projeto contribuiu consideravelmente para a qualificação da assistência prestada e expansão das ações aos usuários em sofrimento psíquico no âmbito da equipe estudada, proporcionando o repensar de paradigmas originários do modelo hospitalocêntrico, aprimorando o conhecimento sobre a temática de saúde mental, e estabelecendo uma ressignificação do olhar voltado ao paciente, com ênfase na construção da autonomia, cidadania e reinserção social.

Apesar das contribuições, o PET-RAPS ainda enfrenta desafios que precisam ser superados para que suas ações sejam mais efetivas. Entre eles, a falta de profissionais na equipe, que é um desafio a ser vencido - porém, o PET-RAPS não tem governabilidade para solucioná-lo sozinho, por ser relacionado a instâncias maiores, como a Secretaria de Saúde Municipal e Prefeitura do município de Cuiabá-MT.

Fica evidente a importância dos projetos de ensino, extensão e de pesquisa para a qualificação da assistência, sendo de grande importância continuar investindo na construção de novas relações ensino-serviço-comunidade, instigando a reorganização e reflexão da prática de saúde mental na atenção básica, gerando sujeitos mais críticos, ativos e com visão diferenciada, capazes de mudar a realidade da saúde mental e da atenção básica, contribuindo para a efetivação dos princípios e diretrizes da Reforma Psiquiátrica e do Sistema Único de Saúde.

Embora este estudo tenha uma importante contribuição na avaliação da repercussão do projeto desenvolvido, algumas limitações se referem: a poucas informações sistematizadas registradas em prontuários, e à escassez de trabalhos que avaliem e discutam a atuação do PET na intersecção entre a ESF e os serviços de saúde mental. Por isso, consideramos fundamental continuar desenvolvendo trabalhos relacionados a essa temática. 


\section{Referências}

1. Amarante PDC. Saúde mental e atenção psicossocial. Rio de Janeiro: Fiocruz; 2007.

2. Brasil. Lei $n^{\circ} 10.216$, de 6 de abril de 2001. Dispõe sobre a proteção e os direitos das pessoas portadoras de transtornos mentais e redireciona o modelo assistencial em saúde mental. Diário Oficial União. 9 abr 2001.

3. Conselho Estadual de Saúde de Mato Grosso. Resolução no 14/2011. [Aprova a Política Estadual de Saúde Mental em Mato Grosso] [acesso 13 nov 2014]. Disponível em: http://www.saude.mt.gov.br/upload/noticia/2/arquivo/191110161618-SES-MT-Acapaconferencia-saude-mental.pdf

4. Ministério da Saúde (BR). Ministério da Educação. Portaria Interministerial n 421, de 3 de março de 2010. Institui o Programa de Educação pelo Trabalho para a Saúde (PET Saúde) e dá outras providências [Internet] [acesso 2014 Dez 5]. Disponível em: http://bvsms.saude.gov.br/bvs/saudelegis/gm/2010/pri0421_03_03_2010.html

5. Ministério da Saúde. Programa de Educação pelo trabalho Pet-Saúde [Internet] [acesso 7 nov 2014]. Disponível em: http://www.portal.saude.gov.br/portal/saude/profissional/visualizar_t. cfm?idtxt $=3530$

6. Silveira DP, Vieira ALS. Saúde mental e atenção básica em saúde: análise de uma experiência no nível local. Ciênc Saúde Colet. 2009;14(1):139-48. http://dx.doi.org/10.1590/S1413-81232009000100019

7. Dalla Vecchia M, Martins STF. Desinstitucionalização dos cuidados a pessoas com transtornos mentais na atenção básica: aportes para a implementação de ações. Interface (Botucatu). 2009;13(28):151-64. http://dx.doi.org/10.1590/S1414-32832009000100013

8. Pinto AGA, Jorge MSB, Vasconcelos MGF, Sampaio JJC, Lima GP, Bastos VC et al. Apoio matricial como dispositivo do cuidado em saúde mental na atenção primária: olhares múltiplos e dispositivos para resolubilidade. Ciênc Saúde Colet. 2012;17(3):653-60. http://dx.doi.org/10.1590/S1413-81232012000300011

9. Rodrigues ES, Moreira MIB. A interlocução da saúde mental com atenção básica no município de Vitória/ES. Saúde Soc. 2012;21(3):599-611.

10. Duarte SJH, Pereira JG, Santos NC, Pereira GAS, Pereira WR. Contribuições do PET-Saúde da família na formação de enfermeiros e médicos no município de Cuiabá, MT. Ciênc Cuid Saúde. 2012;11(4):813-9. http://dx.doi.org/10.4025/cienccuidsaude.v11i4.13600

11. Bardin L. Análise de conteúdo. 3a ed. Lisboa: Edições 70; 2004.

12. Delfini PSS, Sato MT, Antoneli PP, Guimarães P. Parceria entre CAPS e PSF: o desafio da construção de um novo saber. Cienc Saude Colet. 2009; 14 Supl. 1:1483-92.

13. Tanaka OY, Ribeiro EL. Ações de saúde mental na atenção básica: caminho para ampliação da integralidade da atenção. Ciênc Saúde Colet. 2009;14(2):477-86. http://dx.doi.org/10.1590/S1413-81232009000200016

14. Chiavagatti FG, Kantorski LP, Willrich JQ, Cortes JM, Jardim VMR, Rodrigues CGSS. Articulação entre Centros de Atenção Psicossocial e Serviços de Atenção Básica de Saúde. Acta Paul Enferm. 2012; 25(1):11-7. http://dx.doi.org/10.1590/S0103-21002012000100003

15. Souza AC. Estratégias de inclusão da saúde mental na atenção básica no Rio de Janeiro: um movimento das marés [tese]. Rio de Janeiro (RJ): Escola Nacional de Saúde Pública Sergio Arouca; 2012.

16. Ministério da Saúde (BR). Portaria n 336, de19 de fevereiro de 2002. Dispõe sobre a proteção e os direitos das pessoas portadoras de transtornos mentais e redireciona 0 modelo assistencial em saúde mental. Diário Oficial União. 20 fev 2002; seção1. 
17. Correia VR, Barros S, Colvero LA. Saúde mental na atenção básica: prática da equipe de saúde da família. Rev Esc Enferm USP. 2011;45(6):1501-6. http://dx.doi.org/10.1590/S0080-62342011000600032

18. Peduzzi M, Norman IJ, Germani ACCG, Silva JAM, Souza GC. Educação interprofissional: formação de profissionais de saúde para o trabalho em equipe com foco nos usuário. Rev Esc Enferm USP. 2013; 47(4):977-83. http://dx.doi.org/10.1590/S0080-623420130000400029

19. Oliveira AGB, Vieira MAM, Andrade SMR. Saúde mental na saúde da família: subsídios para o trabalho assistencial. Cuiabá: Olho D'Água; 2006.

20. Ministério da Saúde (BR), Secretaria de Vigilância em Saúde. Guia de vigilância epidemiológica. 6a ed. Brasília (DF): MS; 2005.

21. Antunes B, Coimbra VCC, Souza AS, Argiles CTL, Santos EO, Nadal MC. Visita domiciliar no cuidado a usuários em um centro de atenção psicossocial: relato de experiência. Ciênc Cuid Saude. 2012; 11(3):600-4. http://dx.doi.org/10.4025/cienccuidsaude.v11i3.15416

22. Coelho MO, Jorge MSB. Tecnologia das relações como dispositivo do atendimento humanizado na atenção básica à saúde na perspectiva do acesso, do acolhimento e do vínculo. Ciênc Saúde Colet. 2009;14 Supl. 1:1523-31. http://dx.doi.org/10.1590/S1413-81232009000800026

23. Ministério da Saúde (BR), Secretaria de Atenção à Saúde. Saúde mental. Brasília, DF; 2013. (Cadernos de Atenção Básica, n. 34).

24. Campos RO, Trapé TL, Belo KO, Kores RC, Dorigatti AE. O PET-Saúde como instrumento para a articulação da saúde mental e coletiva: narrativas da formação e do trabalho em saúde. Cad Bras Saúde Mental. 2012; 4(8):176-85.

25. Quinderé PHD, Jorge MSB, Nogueira MSL, Costa LFA, Vasconcelos MGF. Acessibilidade e resolubilidade da assistência em saúde mental: a experiência do apoio matricial. Ciênc Saúde Colet. 2013;18(7):2157-66. http://dx.doi.org/10.1590/S1413-81232013000700031

Rézio LA, Moro TN, Marcon SR, Fortuna CM. Contribuciones del PET-Salud/Redes de Atención Psicosocial en la Salud de la Familia. Interface (Botucatu). 2015;19 Supl 1:793-804.

Este estudio tuvo como objetivo analizar las contribuciones del Programa de Educación en el Trabajo para la Salud - PET-Salud/Redes de Atención Psicosocial en la Salud - de una Unidad de Estrategia de Salud de la Familia en Cuiabá, Mato Grosso, Brasil. Se trata de un estudio descriptivo, con abordaje cualitativo, a través de entrevistas de cinco profesionales de la salud y dos estudiantes becados y los registros de actividad utilizadas en servicio. A la luz del análisis de contenido temático hemos sistematizado cuatro subtemas: "Articulación de la red"; "Calificación de la atención de la salud mental", "mejora del conocimiento de los profesionales" y "la superación del estigma del sufrimiento psicológico del paciente". Hubo una conciencia de los profesionales y estudiantes sobre la importancia del seguimiento de personas con enfermedad mental en la atención primaria con relación a los servicios de salud mental.

Palabras clave: Salud mental. Desarrollo Personal. Atención Primaria de la Salud. Trabajadores del Sector Salud. Programa de Educación en el Trabajo para la Salud. 
